\title{
Autonomous coordination of heterogeneous vehicles for persistent monitoring problem with route and fuel constraints
}

\author{
Maksim Kenzin, Igor Bychkov and Nikolai Maksimkin \\ Matrosov Institute for System Dynamics and Control Theory of Siberian Branch of Russian \\ Academy of Sciences (IDSTU SB RAS), Irkutsk, Russia \\ E-mail: gorthauers@gmail.com
}

\begin{abstract}
The problem considered in this paper is to produce both routes and refuelling schedules for a heterogeneous fleet of autonomous mobile vehicles that provide efficient group work performance in large-scale persistent monitoring missions. In the model, the route for the group of vehicles must be determined on the extended time period to ensure regular and well-timed inspections of a set of tasks at scheduled intervals. The routes are limited by the speed limitation, onboard equipment and fuel capacity of the vehicles alongside with visitation frequency and equipment requirements of each task. Fuel capacity limitations force vehicles to recharge (refuel) at intervals using charging stations within the operation area. It is a problem of considerable practical interest to effectively route the heterogeneous group of vehicles under continuous rotation and in a dynamic environment with vehicle systems being subjected to malfunctions and unforeseen events. We propose two-level architecture for the group control system to coordinate cooperative vehicle actions during long-term multi-objective missions: while the high-level planner is designed to dynamically adjust fail-safe refuelling schedules for the vehicles in the group, the low-level planner is aimed to the accurate generation of the local group routes ensuring well-timed accomplishment of all tasks under technical constraints.
\end{abstract}

\section{Introduction}

The rapid evolution of the robotics technologies in recent years has led to the significant reliability improvement of research, military, and commercial autonomous vehicle systems. This has encouraged new trends in environmental robotics science relating to robot and sensor network interaction, model-aided path planning, adaptive sampling, and cooperative robotic teams. In situations when it is required to establish a sampling network within the certain region in short order (militaristic operations, natural disasters, etc.), using a team of autonomous mobile robots (AMR) allows organizing an efficient large-scale environmental monitoring of areas of interest.

However, a large range of fundamental problems needs to be solved in order to achieve highperformance cooperative teamwork in a hostile and ever-changing environment [1]. Dynamic mission and task planning problem for the large cooperative robot teams is one of the most essential research challenges among these as it allows more optimal resource utilization, particularly within coordinated heterogeneous systems.

Copyright (C) 2019 for this paper by its authors. Use permitted under Creative Commons License Attribution 4.0 International (CC BY 4.0). 
In general, the mission-planning problem for the environmental monitoring is a variation of vehicle routing problem (VRP) under specific spatio-temporal constraints: a set of different tasks should be feasibly allocated between vehicles of the group in the most efficient visitation order. In the context of persistent monitoring, when the robot team is deployed in an environment over a sustained period of time, mission tasks usually require not the single visitation but the series of periodic inspections by AMRs with a given frequency [2].

A significant limitation in the aspect of long-term planning is the periodic refuelling necessity due to the limited AMRs battery capacity [3]. Thus, a fully autonomous control system must not only provide efficient group route planning but also regulate the refuelling process in the course of the mission under low-communication bandwidth. Such two-level planning procedure for the group strategy should be both flexible and reliable at the same time to ensure the prompt reaction to any unforeseen events and unplanned conditions changes. In this regard, the intelligent control system development for the heterogeneous group of AMRs comes to the fore.

\section{Problem formulation}

In general, persistent monitoring missions require a group of vehicles to visit and inspect (perform some research activities) the set of tasks at scheduled intervals on an extended time period and under operational requirements. The routing problem here is to find a feasible group route ensuring, as far as possible, the well-timed inspection of the majority of tasks under continuous change of currently working team due to the robots recharging needs. The problem parameters are defined as follows below.

We denote by $T$ the whole mission length and suppose that it is a big value in the context of global mission planning. Assume there is a set of tasks $N=\{1, \ldots, n\}$ distributed over a specific operational area. These tasks are defined not only by their location in space but also with the demanded periodicity of inspections $p_{i}$ and the single-inspection duration time $s_{i}, i \in N$, which all are known in advance. On top of that, a specific type of research equipment $e_{i} \in\{1,2, \ldots, l\}$, $i \in N$ is required for the inspection of each task.

The periodicity value $p_{i}$ defines the demanded duration of the time interval between two successive inspections of $i$-th task by robots of the group. Hence, in case of arrival ahead of time, AMRs should loiter until the expiration of the required interval $p_{i}$, and, in case of delayed inspection, new period $p_{i}$ for the $i$-th task will start not from the expected, but from the actual inspection time.

The group of robots performing the mission consists of $m$ vehicles, which may differ by their cruising speed $v^{k}$, battery capacity $b^{k}$, battery level $b(t)_{c}^{k} \leq b^{k}$ and set of onboard equipment $d_{j}^{k} \in\{0,1\}, j=1, \ldots, l, k=1, \ldots, m$, where $l$ is the number of all types of research equipment. Battery limitations force AMRs to refuel at intervals by docking to the specialized charging stations. Concrete locations of these are not essential since we only need the estimated travelling time for each robot to get to the nearest free recharging dock from the operation area. We denote the average charging speed for all charging stations by constant parameter $c \geq 1$. In that case, full charging time for empty battery with a capacity of $b$ hours would be $b / c$ hours. Here we rate batteries not in energy units but as average run-time on cruising speed. We assume that the number of stations/docking spaces is not limited so that there is always a free dock for a vehicle in need. Additionally, we assume that each type of equipment is installed on at least two different vehicles. This assumption allows the group to send any single robot to a recharging station without losing the performance capability of the remaining team.

The process of persistent monitoring mission implementation in the statement proposed above is schematically shown in the figure 1. At the depicted time point, the currently working team consists of two unmanned vehicles; one more vehicle has recently left the group and now is travelling to the charging station for refuelling; the fourth vehicle has already been refuelled and now it is on its way to re-join the working team at the specified rendezvous location. 


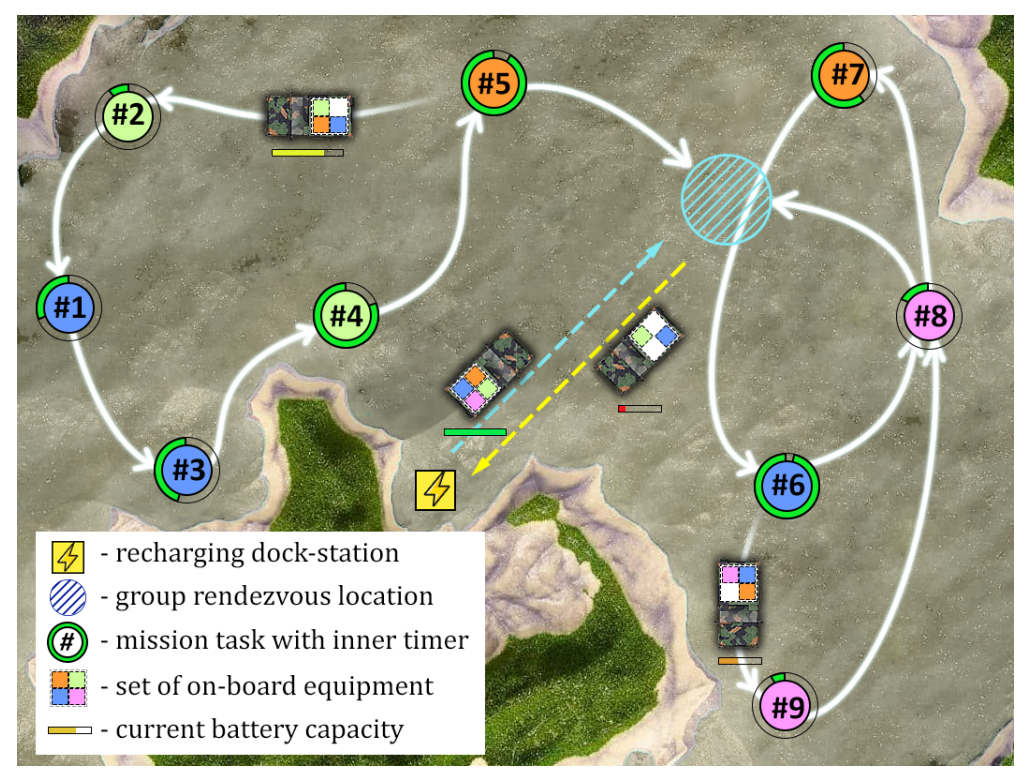

Figure 1. Schematic representation of the persistent monitoring mission for heterogeneous group of four AMRs.

It is worthy of note that the multi-vehicle system should be self-controlled with no human operator or another external supervisor involved. Thus, all parallelizable calculations should be distributed among robots to achieve both maximal processing power and decentralized control hierarchy. In order to achieve proper decentralization, robots in the squad should coordinate in a way to periodically establish group communication sessions for inner-vehicle data transferring. As the real communication channels are typically slow and limited in range, we assume that communication sessions could be established only during so-called rendezvous, when all accessible AMRs simultaneously arrive at the specified location.

The requirement for periodic communication sessions primarily arises due to the dynamic nature of real-world missions when both the working group and set of tasks could be subjected to unexpected changes. These changes may occur in real-time, making it necessary to adjust the current plan in order to maximize the group efficiency in new conditions. Among the events that require re-planning are:

- adding new tasks or withdrawing the old ones;

- change of tasks parameters;

- unexpected AMR loss or malfunction;

- collecting new AMR of previously lost one;

- AMR leaving the group for recharging;

- collecting recently recharged AMR.

The effectiveness of the group as a whole is defined by maintaining the regularity of welltimed inspections of all tasks. Situations of vehicles arriving too late and delaying the inspection are undesirable and should be excluded, if possible. Thus, our objective is to develop the synchronous control architecture able to construct and adjust group routes providing: minimum inspection delays during continuous persistent monitoring; effective management of AMRs refuelling process; regular communications sessions within the group. 


\section{Problem classification}

The proposed problem combines features of a number of well-known group routing problems, all of which are NP-hard: coverage and patrol routing problem, multiple travelling salesman problem (mTSP) and several variations of the classical vehicle routing problems (VRP). The formulation of the vehicle routing problem and some methods for its solution were first proposed in [4]. In the classical VRP, the purpose of the vehicles is to serve a set of customers with known demand by delivering goods from the warehouse (depot), while the routing problem itself is to find the optimal group route with respect to the given criterion (time, distance, etc.). The most significant VRP variations are VRP with time windows (VRPTW), which utilize service-time requirements of customers, heterogeneous fixed fleet vehicle routing problem (HFVRP) with functionally diversified vehicles, and a green VRP (g-VRP), where vehicles with limited driving range should additionally consider refuelling activities. In cases where the actual delivery process is absent, these problems are reduced to the mTSP where the shortest loop trajectories should be constructed for the group of agents to visit all the customers under spatio-temporal constraints.

In coverage and patrol problem a group of agents should continuously revisit a set of waypoints within a given area in order to maximize the visit frequency for each waypoint [5]. Most commonly, these waypoints are located in a way to ensure full coverage of the patrol area. The extended formulation of the patrolling problem with intrusion prevention is studied in [6]: a graph of potential targets is given and the time needed by the intruder for a successful strike is assigned to each target. Thus, the routing problem is to find the group route for the patrolling agents to ensure that each target is visited with a frequency that guarantees intruder detection.

The nature of the persistent visitation problem presented in [7] lies at the intersection of the patrolling problem and g-VRP. Firstly, there is no planning horizon in the ordinary sense in [7]. Instead, each target is characterized by the minimal required visitation frequency. Secondly, vehicles are limited by fuel capacity and are able to refuel at the specialized stations located within the mission area for predetermined prices. The problem is to construct a feasible route ensuring an infinite sequence of visitations such that no visitation is ever overdue at the lowest refuelling cost. The persistent visitation problem is proved to be NP-complete as a new original VRP variation even for a single vehicle.

The problem of persistent visitation shares a lot of features with the persistent monitoring problem proposed in this work. At the same time, there are significant differences between two problems that do not allow us either to classify them as variations of the same problem nor to use identical methods and ideas to solve them. The main difference here is the target service-time requirements: while in [7] they are similar to the soft time window constraints from VRPTW and as visitations ahead of time are allowed, in this work we consider that the vehicles always have to wait exactly for the required time interval, which is closer to the hard time window constraints. It is worth noting that soft time requirements alongside with static conditions allow authors of [7] to construct the solution as an infinite sequence of identical cycles, where each cycle is a sequence of visitations starting and ending at the same target such that all other targets have been visited.

In the scientific literature, there is a large number of works that explore different extensions of the persistent visitation model. However, the overwhelming majority of them are devoted to constructing cyclical trajectories of limited length. Restrictions of the hard type do not allow such mission decomposition, which leads us to the acyclic routes with a floating planning horizon [8]. The authors of this work are not aware of group monitoring models that combine hard time window restrictions with periodic refuelling, group heterogeneity, and inner-vehicle communication needs due to the dynamic mission conditions. Such routing problems utilizing a whole range of various constraints and requirements are commonly referred to in modern scientific literature as a wide class of rich vehicle routing problems [9]. 


\section{Two-level control system}

Effective mission planning for real-world robotic operations is a complicated and challenging problem, especially when it is required to respond to environmental changes and unexpected events. While even in static and fully known environment group routing alone is a problem of high computational complexity, dynamic conditions drastically devalue the requirement for an optimal solution [10]. In this regard, we propose the two-level approach allowing to perform continuous persistent monitoring. The proposed approach utilizes an online mission decomposition on the upper-level that provides both communication accessibility and computational load reduction, while lower-level route planner is required to handle graph search constraints and carry out the task assignment. Ideally, mission decomposition points should be associated with essential condition changes as the low-level routing for each operating period (the period between two consecutive decomposition points) in this case would be treated as static. Nevertheless, as we can predict reliably only those events that relate to AMRs refuelling needs, we propose the following mission decomposition scheme based on the expected vehicle rotation (figure 2 ).

Rendezvous points (AMR s leaving and re-joining the group for/after refuelling)

\begin{tabular}{|c|c|c|c|c|c|}
\hline Operating Period \#1 & OP \#2 & OP \#3 & OP \#4 & OP \#5 & \\
\hline \multirow{3}{*}{ Mission Start } & & & & & \\
\hline & AMR "A" & & & AMR “C” & \\
\hline & Refuelling & AMR “B” & & & AMR “D” \\
\hline
\end{tabular}

Figure 2. Mission decomposition on the basis of vehicle squad rotation.

According to this scheme, each decomposition point triggers a rendezvous of the currently working AMRs for the purpose of both communication and vehicle rotation activities due to the refuelling needs (sending robots to the recharging station or collecting recently recharged vehicles back to the group). As the rejuvenated group continues the task's inspection until the next decomposition point, each vehicle in the group computes to find the best group route for the next operating period considering the upcoming group rearrangement: which AMRs would leave the group during next rendezvous and which would rejoin. Such a pre-planning would allow for saving time and shortening the rendezvous length alongside with calculations distribution among vehicles. In such an instance, inner-vehicle communication at the end of each operating period should include the exchange of the AMR's best-found solutions (figure 3).

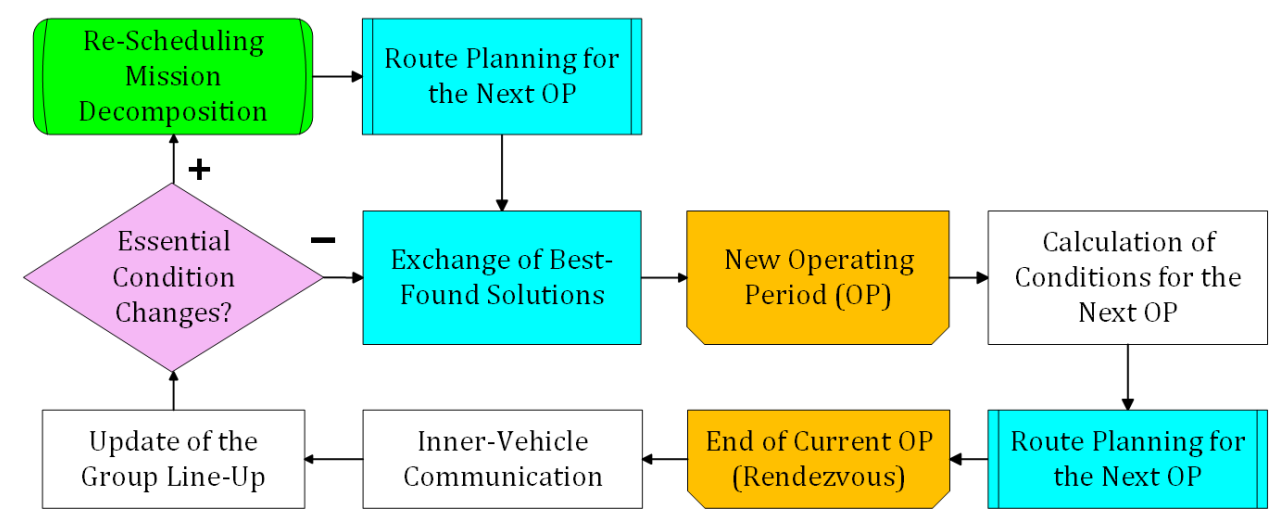

Figure 3. Scheme of two-level group control system. 
Consequently, the upper-level mission planner strategy is to manage operating periods by scheduling AMRs recharging cycles. A mission planner should be simple, fast and reliable, as quick and effective re-planning is required each time an unexpected event occurs. On the other hand, the lower-level route planner is not designed to react to environmental dynamic changes. However, as the operating field is split via decomposition to the smaller spaces, the routing system should be capable of high-performance local group motion planning under spatiotemporal constraints. The routing problem here is to achieve the well-timed task's inspection for the group of functionally heterogeneous robots and to ensure simultaneous group arrival at the rendezvous location by the end of the operating period. Task allocation problem on a single operating period should not be treated separately from the global objective as successive operating periods share common terminal conditions. As both global mission planning and local route planning are essential for monitoring efficiency, proper cooperation and synchronization between both levels would allow the control system to deal with complex requirements and restrictions, which are aimed at the more accurate simulation of real-world problems [11].

\subsection{Mission planner}

Summarizing the above, the high-level mission planner is to construct an appropriate group rotation schedule providing reliability and high-performance capability of the working group. As mission length $\mathrm{T}$ is supposed to be a considerable big value, we suggest using search space discretization to speed up both encoding and finding the solutions. For that reason, we will consider mission time $T$ as a sequence of equal time intervals $T=\left\langle T^{1}, \ldots, T^{z}\right\rangle, z=T / T_{0}$, where $T_{0}$ is the duration of each interval. In this representation, the working schedule of a single robot can be denoted by a binary $z$-dimensional vector, where each attribute $h$ stands for a robot's status during the corresponding time interval $(h=0$ if the vehicle is currently working and $h=1$ otherwise). A schedule of the $i$-th vehicle is considered as feasible if none working period (continuous sequence of $x$ 0-attributes) lasts longer than the vehicle's battery level at the beginning of that period $x \cdot T_{0} \leq b\left(t_{x}\right)_{c}^{i}$. The group schedule at this point can be represented as $z$-by- $m$ binary matrix $H=\left\{h_{i j}\right\}$ as shown in the figure 4 .

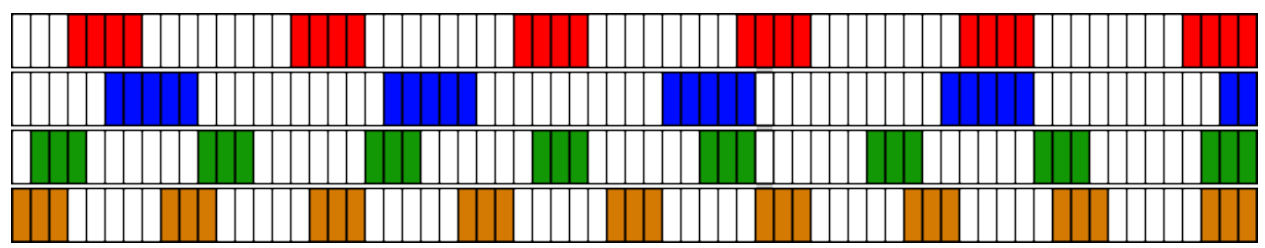

Figure 4. Group schedule. Coloured cells represent charging periods of robots.

As AMRs are not obliged either to leave the group only being completely discharged nor to always recharge up to the entire full battery (although it is preferable), we can adjust charging periods for each robot during the mission in order to obtain the desirable group rotation schedule:

(1) The first and the main requirement is to maintain robots in the group in good working order by organizing well-timed recharging for all vehicles in need.

(2) Secondly, the working group should always be functionally able to inspect each mission task by having access to all available types of onboard equipment.

(3) Then, we want to exclude as far as possible the simultaneous mass charging of several robots since it reduces the performance capability of the remaining group.

(4) Finally, since each rendezvous distracts robots from the task's inspection, we want to exclude excessive rendezvous where possible. In order to do this, we want to conjoin closely-spaced rendezvous together by shifting them to the common time-point. 
We regard the first requirement from the list above as a feasibility criterion and the next three as efficiency criteria for the planning problem. Thus, we propose using a convolution of two different functions as the global objective function here:

$$
f(H)=f_{G}(H)+z \cdot f_{R}(H) \rightarrow \min
$$

The first function $f_{G}(H) \in[0, z]$ evaluates given schedule $H$ in respect of the group performance capability during the mission progress, while the second one $f_{R}(H) \in[0,1]$ keeps track of the rendezvous frequency. The loss function (2) evaluates the performance capability of absent robots on each interval $T^{i}$ of the mission by using three different multiplier factors responsible for the number, speed of recharging robots and equipment availability, respectively:

$$
f_{G}(H)=\sum_{i=1}^{z}\left(\left(\sum_{j=1}^{m} h_{i j}\right) \cdot\left(\frac{\sum_{j=1}^{m} h_{i j} v^{j}}{\sum_{j=1}^{m} v^{j}}\right) \cdot\left(1-\prod_{k=1}^{l}\left(1-\frac{\sum_{j=1}^{m} h_{i j} d_{k}^{j}}{\sum_{j=1}^{m} d_{k}^{j}}\right)\right)\right)
$$

On each time period $T^{i}$ function (2) takes the value on the interval $[0,1]$. More specifically, 0 value means that all robots are currently working in the group and 1-value stands for undesirable situations when all carriers of the particular equipment are currently recharging.

The second objective function estimates the number $F_{E}(H)$ of expected rendezvous (time intervals, where at least one vehicle is changing its status) and compares it to the minimum possible number of rendezvous $F_{I}(H)$ :

$$
\left.f_{R}(H)=\frac{F_{E}(H)-F_{I}(H)}{F_{E}(H)}, F_{E}(H)=\sum_{i=2}^{z}\left(1-\prod_{j=1}^{m}\left(1-\left|h_{i j}-h_{i-1 j}\right|\right)\right)\right), F_{I}(H)=m \cdot T / \sum_{j=1}^{m}\left(b^{j} / c\right)
$$

Since function (3) always takes the value on the interval $[0,1]$, we use weighting factor $z$ in the objective function (1). Thus, the planning problem is to find such feasible schedules, which would satisfy objective function (1) the most. The feasibility of a schedule as a whole cannot be determined in the explicit form and so it should be checked additionally via specialized algorithmic procedure considering such AMR's parameters as speed, battery level, and capacity alongside with travelling time to reach the nearest charging station and, finally, charging rate.

In general, the scheduling problem is NP-complete, and therefore the use of heuristic methods to solve it seems the most reasonable. Thus, we propose using a simple modification of genetic algorithm (GA) since GA offer near-optimal solutions in a faster time and with better scalability at average than other heuristic and meta-heuristic approaches [12]. GAs are mostly appropriate for large-sized high-dimensional problems but may converge to local optima in a finite time.

To decrease the problem size we use a compressed vector representation of the matrix-schedule $H$ as the chromosome (figure 5) with function (1) as the objective function. To define the chromosome's fitness, the matrix form of the solution should be restored at first by specialized repairing procedure that combines solution decoding with intelligent local optimization to decrease the number of possible rendezvous. For the genetic operators, we suggest using random mutation with both two-point and PPOX-crossovers as they are widely recognized to be the most efficient genetic operators for a variety of scheduling problems.

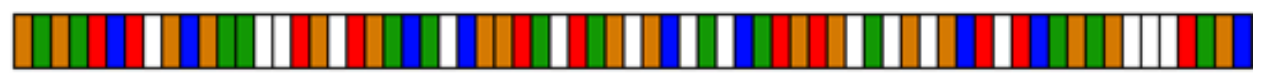

Figure 5. One-dimensional representation of the group schedule from figure 4 . 


\subsection{Route planner}

High-level mission decomposition allows us to eliminate refuelling aspect of the problem and neutralize the ever-changing group composition challenge for the route planning problem. Besides, it leads to the problem space reduction, which is another advantage of the proposed approach. The routing problem on a single operating period is formulated as stated above in Section 2. Status of each object of the mission is inherited from the previous operating period, a subset of $k \leq m$ currently working robots is located within the rendezvous area and each vehicle is guaranteed to have enough battery level to work through the whole operating period.

Let us denote by $r$ the route of a single robot as a list of task numbers in the consecutive order of their planned visits:

$$
r=\left\langle V_{0}, V_{1}^{r}, V_{2}^{r}, \ldots, V_{u}^{r}, V_{0} .\right\rangle
$$

As can be seen, vehicles start each period from the rendezvous point $V_{0}$ and travel back there at the end of the period to take part in group rendezvous. It also should be noted that any task could be included more than once into the route of a single robot. The group route is a set of all single AMRs routes (4):

$$
R=\left\{r_{1}, \ldots, r_{k}\right\} .
$$

The routing problem is to generate feasible group route (5) providing:

- regular and well-timed inspections of all tasks;

- on-time arrival of all vehicles to the rendezvous location;

- serviceable mission conditions at the end of the operating period (no tasks with expired or near-expired inspections).

To measure the quality factor of different group routes in terms of given requirements, we propose a scenario-based approach that relies on the list of desirable and undesirable both singlerobot and group behaviours. Each scenario includes a priority-order value, set of triggering events, scoring type (penalty or reward) and scoring conditions. Trigger events of all scenarios are checked via the simulation run of the group work with the output being a sum of reward and penalty points weighted according to given priorities. For scaling and normalizing points charged by different events, we use an original scoring technique presented in our earlier works. This technique introduces an additional hotness function $a_{i}^{k}(t)$ evaluating the relevance of each task inspection by a given robot at any moment of time. Hotness function is constructed in such a way, that in the case when the group is unable to inspect all tasks without any delays, well-timed inspections would be assured for tasks with lesser periodicity, which are likely to be more important. Further particular details of the normalizing procedure can be found in $[10,13]$.

Turning back to the routing problem studied in this work, we suggest using the next four scenarios presented in table 1.

Table 1. List of scenarios for a group persistent monitoring mission.

\begin{tabular}{llll}
\hline Priority & Trigger Event & Type & Scoring Conditions \\
\hline$\# 1$ & Each time vehicle visits a task & Penalty & Lack of required equipment \\
$\# 2$ & Each time vehicle starts inspection & Penalty & Inspection is delayed \\
$\# 3$ & Group arrives at the rendezvous location & Penalty & Bad future conditions \\
$\# 4$ & Group arrives at the rendezvous location & Penalty & Timely disunited arrival \\
\hline
\end{tabular}


The lower-level route planning problem is a much more complicated and computationally intensive problem as it is known to be NP-hard. Thus, there are no algorithms solving it in polynomial time, which leads us to the class of approximation algorithms which allow obtaining rational sub-optimal solutions in low computational time. Over the past decade, heuristic and meta-heuristic approaches for solving the routing problems have been rapidly developing. So far, the most efficient methods apply decomposition schemes, intelligent neighbourhood search strategies and components of exact optimization methods. Newly designed heuristics also became more flexible and versatile as they could be applied to a wide range of VRP variations without any structural changes.

Among others, evolutionary methods have proven to be highly successful both on a classical VRP and on a number of its variations [14]. The main advantage of evolutionary algorithms (EAs) is the ability to find solutions for high-dimensional poorly structured problems and problems with complex constraints, as EAs require a relatively small amount of information about the nature of the problem. Thus, we propose a hybrid evolutionary approach featuring specialized genetic operators, advanced local search heuristics and solution improvement techniques to address both the expectable large-size of the problem and complex spatio-temporal constraints. Both the algorithm's structure and original heuristics are in-depth studied in our works $[13,15]$ devoted solely to the low-level routing problems for both homogeneous and heterogeneous groups of vehicles, so we omit the details here.

\subsection{Experimental results}

Both mission- and route- planning algorithms are implemented in our simulation framework "Multiobjective Mission Planner" to run a series of simulation studies. The high efficiency of the suggested approach is shown through these tests. Upper-level mission planner is proved to be fast, accurate and reliable as it allows fast construction of group schedules within the $3 \%$ of the optimal value (figure 6). Lower-level routing system (figure 7), in return, offers efficient near-optimal $(\leq 1 \%)$ task allocation procedure even under strict constraints and the large-sized search field. The proposed cooperation scheme between both planners allows the group to be efficient under continuous squad rotation due to the recharging needs.

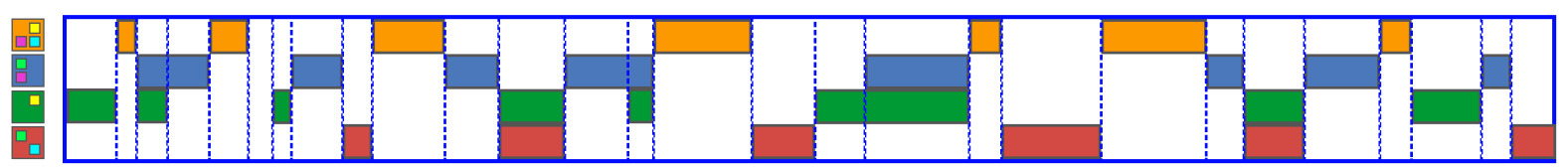

Figure 6. Recharging schedule for the group of four functionally heterogeneous vehicles.

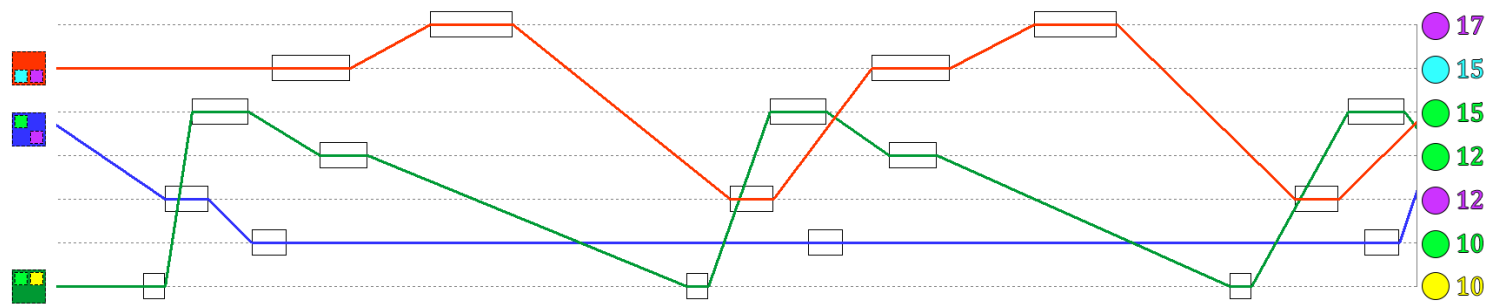

Figure 7. Group route for three heterogeneous AMRs over seven tasks of different periodicity. 


\section{Conclusion}

This paper presents a two-level approach to the AMRs persistent routing problem that incorporates rendezvous point-selection scheme providing advantageous mission decomposition and local task allocation scheme to coordinate currently available heterogeneous vehicles in the most efficient way. The next stage of this work involves a wide diversity of mission tasks: ones that couldn't be inspected by a single robot, tasks with inspection time relying on the number of inspecting vehicles, prolonged and areal task's positions, etc. Another extension of this work is to develop a real-time path planner to deal with complex and unfamiliar environments, collision avoidance and dynamic obstacles, as an additional, third level of the group control system.

\section{Acknowledgments}

This work was supported by the Russian Science Foundation, project no. 16-11-00053-П. This work was also supported in part by the Presidium RAS, program no. 7, project "Methods, algorithms and tools for the decentralized group solving of problems in computing and control systems" (reg. no. AAAA-A18-118031590006-2).

\section{References}

[1] Dunbabin M and Marques L 2012 Robots for environmental monitoring: significant advancements and applications IEEE Robotics \& Automation Magazine 19(1) 24-39

[2] Smith R N, Schwager M, Smith S L, Jones B H, Rus D and Sukhatme G S 2011 Persistent ocean monitoring with underwater gliders: Adapting sampling resolution J. Field Robotics 28 714-41

[3] Afshar-Bakeshloo M, Mehrabi A, Safari H, Maleki M and Jolia F 2016 A green vehicle routing problem with customer satisfaction criteria $J$. of Industrial Engineering International 12(4) 529-44

[4] Christofides N 1976 The vehicle routing problem Revue Française d'Automatique, Informatique, et Recherche Opérationelle 10(2) 55-70

[5] Chevaleyre Y 2004 Theoretical analysis of the multi-agent patrolling problem Proc. of the IEEE/WIC/ACM Int. Conf. on Intelligent Agent Technology pp 302-08

[6] Basilico N, Gatti N and \& Villa F 2010 Asynchronous multi-robot patrolling against intrusions in arbitrary topologies Proc. of the 24th AAAI Conf. on Artificial Intelligence pp 1224-29

[7] Fargeas J L, Hyun B, Kabamba P and Girard A 2013 Persistent visitation under revisit constraints 2013 Int. Conf. on Unmanned Aircraft Systems pp 952-57

[8] Stump E and Michael N 2011 Multi-robot persistent surveillance planning as a vehicle routing problem 2011 IEEE Int. Conf. on Automation Science and Engineering pp 569-75

[9] Hartl R F , Hasle G and Janssens G K 2006 Special issue on rich vehicle routing problems Central European J. of Operations Research 14(2) 103-04

[10] Kenzin M, Bychkov I and Maksimkin N 2018 An approach to route underwater mobile robots under continuous squad rotation 41th Int. Conv. on Information and Communication Technology, Electronics and Microelectronics pp 973-78

[11] Zadeh S M, Powers D M W and Yazdani A M 2016 Development of an autonomous reactive mission scheduling and path planning (ARMSP) architecture using evolutionary algorithms for AUV operation in a sever ocean environment Computing Research Repository

[12] Kamal A M, Ikramullah S, Kousar R, Ahmad R, Agha M, Faping Z, Anjum N and Asgher U 2018 Recent research trends in genetic algorithm based flexible job shop scheduling problems Mathematical Problems in Engineering $021-32$

[13] Kenzin M, Bychkov I and Maksimkin N 2015 Hybrid evolutionary approach to multi-objective mission planning for group of underwater robots Mathematical Modeling of Technological Processes 549 73-84

[14] Vidal T, Crainic T G, Gendreau M and Prins C 2013 A hybrid genetic algorithm with adaptive diversity management for a large class of vehicle routing problems with time-windows Computers \& Operations Research 40 475-89

[15] Kenzin M, Bychkov I and Maksimkin N 2018 Task allocation and path planning for network of autonomous underwater vehicles Int. J. of Computer Networks \& Communications 10(2) 33-42 